\title{
Subnotificação da Mortalidade Materna em Campinas: 1992 a 1994.
}

\author{
Underreporting of Maternal Mortality in Campinas from 1992 to 1994.
}

Mary Angela Parpinelli, Aníbal Faúndes, José Guilherme Cecatti, Fernanda Garanhani de Castro Surita, Belmiro Gonçalves Pereira, Renato Passini Júnior, Eliana Amaral

\section{RESUMO}

\begin{abstract}
Objetivos: identificar e investigar a subnotificação da mortalidade materna, a partir das declarações de óbito (DO) com causa presumivel para morte materna em mulheres de 10 a 49 anos, residentes em Campinas, São Paulo, no periodo de 1992 a 1994.

Métodos: foram selecionadas, dentre as 1032 DO de mulheres com idade entre 10 e 49 anos, 216 cuja causa de morte era associada à gravidez declarada ou presumivel. Procedeuse então à busca ativa da causa materna em prontuários clínicos, serviço de verificação de óbito e domicílios.

Resultados: foram identificados oito casos adicionais de morte materna dentre as 204 DO com causa materna presumivel, o que correspondeu a uma subnotificação de $40 \%$ ou a um fator de correção de 1,67 sobre a RMM "oficial". A principal causa de óbito com subnotificação (71,5\% ou 5/ 7 casos) correspondeu a complicações infecciosas do aborto, seguida pela morte materna obstétrica indireta (66,6\% ou $2 / 3$ casos).

Conclusões: a DO não pode ser considerada como única fonte para identificação da morte materna, sendo necessária a investigação complementar das causas presumiveis. A falta de legislação local, além dos aspectos religiosos e sociais, pode estar influindo na omissão do aborto como causa de morte materna.
\end{abstract}

PALAVRAS-CHAVE: Mortalidade materna. Aborto. Saúde reprodutiva.

\section{Introdução}

Até o início desta década estimava-se que 500.000 mulheres morriam no mundo por causas relacionadas ao período gravídico-puerperal ${ }^{1}$. A OMS/UNICEF, em novas estimativas, acrescem a este total cerca de 80.000 mortes maternas anuais, em todo mundo, secundárias à subnotificação de mortalidade e/ou à falha na notificação da causa básica de morte pela declaração de óbito como morte materna ${ }^{2}$. Nesta última condição a causa básica da morte está declarada, entretanto não está declarada a associação com gravidez, parto ou puerpério.

Os países, segundo este estudo, são classificados em cinco grupos: países

Disciplina de Obstetrícia, Departamento de Tocoginecologia Faculdade de Ciências Médicas, Universidade Estadual de Campinas

Correspondência:

Mary Angela Parpinelli

Caixa Postal 6030

13081-970 - Campinas - SP

Fax: (19) 788-9304 desenvolvidos com sistema de registro vital completo e boa atribuição de causa de morte; países desenvolvidos com bom registro de mortalidade, entretanto com deficiente ou nenhuma atribuição de causa básica; países com estimativas de mortalidade materna pelo método RAMOS (inquéritos de mortalidade de mulheres em idade reprodutiva ou "Reproductive Age Mortality Survey"); países com estimativas de mortalidade materna pelo método das irmãs e, por último, países sem estimativas de mortalidade materna.

O método RAMOS preconiza o estudo da mortalidade materna a partir do levantamento do total dos óbitos de mulheres em idade reprodutiva, acompanhado por pesquisa em registros hospitalares, domicílio da mulher e outros meios. Pode ser aplicado aos países com boas estatísticas de mortalidade para identificar os casos de morte materna dentre as mortes presumiveis e, ainda, nos países sem estatísticas de mortalidade ${ }^{2}$.

O Brasil, embora apresente comportamento heterogêneo, tanto do ponto de vista de mortalidade quanto do próprio sistema de informações sobre 
os óbitos, segundo as diferentes regiões da Federação, foi classificado como um país pertencente ao grupo que não possui informações sistematizadas sobre o número de mortes e de classificação da mortalidade materna em suas formas direta e indireta. Segundo esta nova estimativa, baseada na proporção de mortes maternas estimadas para o ano de 1990 e projetadas para o total de mortes de mulheres em idade reprodutiva, houve um acréscimo da ordem de 80 mortes maternas anuais, o que significou um aumento na razão de morte materna de 140 para 220 mortes por 100.000 nascidos vivos ${ }^{2}$.

Para o Estado de São Paulo a Fundação SEADE, órgão oficial para recebimento das notificações de óbito do Estado, considera o sistema de informações sobre mortalidade satisfatório quanto ao registro do número de mortes ocorridas. Contudo, existe significativa porcentagem de óbitos computados como de causa desconhecida ou mal definida. Esta má qualidade da notificação da causa básica do óbito é percebida principalmente para as mortes relacionadas ao ciclo gravídico-puerperal, tornando-a insatisfatória para as estatísticas oficiais de mortalidade materna ${ }^{3,4}$.

Uma das justificativas para a falta de registro adequado da causa de morte de mulheres no ciclo gravídico-puerperal é o despreparo ou a falta de cuidado do profissional médico ao preencher a declaração de óbito, documento oficial para as estatísticas de mortalidade ${ }^{3,5}$. Outras condições, talvez relacionadas a aspectos éticos, religiosos, sociais e até mesmo legais, principalmente para as mortes decorrentes da prática de aborto, podem também estar "mascarando" este aparente despreparo e contribuindo para a omissão do aborto como fator desencadeante dos eventos que levaram à morte ${ }^{6}$.

A grande maioria dos países da América Latina possui leis que restringem a prática do aborto, à exceção de Belize, Cuba e Barbados. Contudo, a existência destas leis não têm sido eficientes para impedir que uma gravidez indesejada termine em aborto induzido ${ }^{7}$. Ao contrário, atribui-se ao aborto ilegal a responsabilidade por até 25 a $50 \%$ das mortes maternas ocorridas, a cada ano, nos países em desenvolvimento ${ }^{8}$.

No Brasil não existem estatísticas oficiais sobre o número de abortos induzidos, nem sequer sobre o número de mortes maternas resultantes de sua prática. Contudo, o Ministério da Saúde estima que são provocados cerca de 1,2 milhões de abortos a cada ano no país ${ }^{9}$. Braga ${ }^{10}$ estimou que $12,7 \%$ das mortes maternas eram causadas por aborto no Brasil dos anos 80. Na cidade de
Recife, em estudo sobre a subenumeração das mortes maternas, os óbitos oriundos das complicações do aborto representaram a segunda maior causa de subnotificação ${ }^{6}$.

Por outro lado, a subnotificação da mortalidade materna como um todo não é exclusiva dos países em desenvolvimento. Países como França, Inglaterra e Estados Unidos, que possuem sofisticados sistemas de registro de mortalidade, também apresentam subnotificação de causas maternas de óbito ${ }^{11,12,13}$. Baseado na possibilidade de morte materna não-declarada, mesmo nestes países desenvolvidos, preconiza-se o ajuste com fator de correção de 1,5 sobre as estatísticas oficiais de mortalidade materna ${ }^{2}$.

$\mathrm{Na}$ certeza da ocorrência de subnotificação das estatísticas oficiais de morte materna na cidade de Campinas, e procurando identificar alguma correlação entre as causas de morte materna e a falha na declaração de óbito, foi proposto este estudo. Os resultados referentes aos óbitos maternos deste estudo encontram-se em outra publicação ${ }^{14}$.

\section{Métodos}

Foram analisadas 1032 DO de mulheres com idade entre 10 e 49 anos, residentes no municipio de Campinas, SP, correspondentes ao total de óbitos femininos em idade fértil ocorridos entre primeiro de janeiro de 1992 e 31 de dezembro de 1994.

A fonte primária das DO foi a Fundação SEADE (Fundação Sistema Estadual de Análise de Dados). A partir da listagem de óbitos, procedeuse à seleção das DO. A etapa seguinte foi a de identificação da causa básica desencadeante do óbito, com reclassificação realizada a partir das informações das DO, segundo a CID (10 a Revisão) ${ }^{15}$. Inicialmente, a identificação destes casos ocorreu classificando-se os óbitos de mulheres em idade fértil em uma das categorias: morte materna declarada (códigos CID O00-O99), presumível e morte não-materna, conforme descrito anteriormente ${ }^{14}$.

Realizou-se posteriormente uma investigação complementar em registros hospitalares e/ou entrevistas com médicos e familiares dos casos cujas causas básicas representassem uma causa de óbito materno declarado e/ou presumivel (método RAMOS). Para este procedimento, os casos foram classificados segundo a procedência da DO (nome do hospital, SVO) e listados. A partir disso, fez-se uma solicitação às diretorias clínicas de todos os hospitais para a pesquisa aos arquivos 
médicos dos prontuários pré-selecionados. Desta forma, foi possivel saber quais casos presumiveis correspondiam realmente a mortes maternas. A soma destes casos às mortes maternas declaradas como tais na DO constituíram as mortes maternas confirmadas.

Não se estudou a mortalidade materna tardia, bem como a morte relacionada à gestação. Esta última categoria inclui o grupo denominado anteriormente como morte não-materna. Calculou-se a razão de mortalidade materna (RMM), tanto bruta ("oficial"), como a corrigida após investigação ("confirmada").

Garantiu-se o sigilo das fontes de informação, sem identificação dos sujeitos, profissionais e hospitais que atenderam ou preencheram as DO. O projeto do estudo foi previamente aprovado pelo Comitê de Ética da instituição.

\section{Resultados}

A Tabela 1 mostra que, em aproximadamente um quinto das declarações de óbito de mulheres em idade reprodutiva, a causa básica declarada não permitiu excluir uma causa materna de morte.

As complicações do aborto tiveram o maior indice de subnotificação $(71,5 \%)$, seguidas pelas causas indiretas de morte materna, subnotificadas em $66,6 \%$ dos casos. A DO mostrou-se satisfatória para a identificação das mortes maternas por complicações tardias da gravidez, do parto e do puerpério, em 90\% dos casos (Tabela 2).

A septicemia ou choque séptico foi a causa declarada de morte que mais ocultou a morte materna por aborto. Entretanto, outros diagnósticos como infarto agudo do miocárdio e broncopneumonia apresentaram como causa básica uma complicação infecciosa de aborto. As causas declaradas de morte estiveram corretas nos dois casos de morte materna indireta, contudo não se declarou o estado grávido-puerperal da mulher (Tabela 3).

As mulheres que tiveram aborto infectado

Tabela 1 - Classificação das mortes maternas, segundo causas básicas registradas na DO - Campinas - 1992 a 1994

\begin{tabular}{lrr}
\hline Classificação & $\mathbf{n}$ & \multicolumn{1}{c}{$\%$} \\
\hline Morte materna declarada & 12 & 1,2 \\
Morte materna presumível & 204 & 19,8 \\
Morte não-materna & 816 & 79,0 \\
Total & 1032 & 100,0 \\
\hline
\end{tabular}

Tabela 2 - Classificação da mortalidade materna pelo grupo de causas, segundo subnotificação e após busca ativa. Campinas, 1992-1994.

\begin{tabular}{lccc}
\hline Classificação & $\begin{array}{c}\text { Casos } \\
\text { subnotificados }\end{array}$ & $\begin{array}{c}\text { Total casos } \\
\text { após estudo }\end{array}$ & $\begin{array}{c}\% \text { de } \\
\text { subnotificação }\end{array}$ \\
\hline MM direta (aborto) & 5 & 7 & 71,5 \\
MM indireta & 2 & 3 & 66,6 \\
MM direta (exceto aborto) & 1 & 10 & 10,0 \\
Total & 8 & 20 & 40,0 \\
\hline
\end{tabular}

Tabela 3 - Distribuição dos casos de morte materna subnotificados segundo o diagnóstico final do estudo e a DO oficial (CID - 10) - Campinas, 1992-1994.

\begin{tabular}{ll}
\hline Diagnóstico final & Diagnóstico original - DO \\
\hline Morte materna direta & \\
Aborto infectado (O08) & Choque séptico (A40 - A41) \\
& Broncopneumonia (J18) \\
Infecção puerperal (O86) & Pulmão de choque (J80) \\
Morte materna indireta & (Hematoma retroperitoneal) \\
Rotura aneurisma cerebral (I60) & \\
\hline Total & AVCH (I61) \\
\hline
\end{tabular}


como causa de morte subnotificada não eram naturais de Campinas, em sua maioria não possuíam seguro saúde e não exerciam trabalho remunerado, ao contrário das que morreram por complicações de doenças prévias (MM indireta). Em todos os casos subnotificados o óbito ocorreu em ambiente hospitalar (Tabela 4).

Tabela 4 - Características sociais das mulheres cujas mortes foram subnotificadas, segundo classificação da morte materna por grupo de causas - Campinas - 1992 a 1994.

\begin{tabular}{|c|c|c|c|}
\hline Características & $\begin{array}{c}\text { MM direta } \\
\text { aborto } \\
\text { n (\%) }\end{array}$ & $\begin{array}{c}\text { MM direta } \\
\text { inf. puerperal } \\
n(\%)\end{array}$ & $\begin{array}{c}\text { MM } \\
\text { indireta } \\
n(\%)\end{array}$ \\
\hline \multicolumn{4}{|l|}{ Faixa etária } \\
\hline $15-19$ & - & - & $1(50 \%)$ \\
\hline $20-24$ & - & $1(100 \%)$ & - \\
\hline $30-34$ & $4(80 \%)$ & - & $1(50 \%)$ \\
\hline [Naturalidade] - Campinas & - & $1(100 \%)$ & $2(100 \%)$ \\
\hline [S. marital] - Sem parceiro fixo & $1(20 \%)$ & - & $1(50 \%)$ \\
\hline [Atividade laboral] - do lar & $4(80 \%)$ & $1(100 \%)$ & - \\
\hline
\end{tabular}

\section{Discussão}

Os resultados mostraram que as causas de morte registradas na DO de mulheres em idade reprodutiva não permitem identificar a causa básica materna em aproximadamente $20 \%$ dos casos. Tal situação confirma a necessidade da melhora na qualidade do preenchimento deste instrumento, que é a fonte primária das estatísticas de mortalidade, não apenas materna mas também da mortalidade geral. Esta situação não é recente. Já em 1987, Laurenti e Mello Jorge ${ }^{5}$, em reedição do texto "Atestado de Óbito", chamam a atenção para aspectos relacionados à qualidade do preenchimento da declaração, destacando, dentre outros, a escolha correta da causa básica da morte.

Diante deste contexto, como era de se esperar, as estatisticas oficiais de mortalidade materna foram subestimadas. No periodo do estudo (1992 a 1994), a RMM oficial para mulheres residentes na cidade de Campinas foi de 25,3 mortes maternas para 100.000 NV (12/47.427). Os atuais resultados identificaram uma RMM confirmada de 42,2 mortes maternas por 100.000 NV (20/47.427), que correspondeu a 40\% de subnotificação de casos ou, ainda, a um fator de correção de 1,67 sobre a RMM oficial. Tal porcentagem de subregistro não diferiu grandemente daquelas encontradas previamente para a cidade de Campinas ou em dados recentes para o município de São Paulo. No primeiro, foi de $37,1 \%$ para o período de 1985 a 1991 e, no segundo, de $40,5 \%$ para o ano de $1998^{4,16}$.

Classificando-se as mortes maternas subnotificadas pelos grupos de causas obstétricas diretas e indiretas, verifica-se maior indice de subnotificação $(71,5 \%$ ou $5 / 7$ casos) para o primeiro grupo, relacionadas às complicações infecciosas do aborto. Para as demais causas obstétricas diretas de morte (excluindo-se o aborto), a subnotificação encontrada foi de $10 \%$ ou $1 / 10$ casos. Esta pequena porcentagem de subnotificação em mortes maternas relacionadas às complicações da gravidez, parto e puerpério, à exceção do aborto, contradiz a tão divulgada falta de preparo do médico para registrar a causa básica do óbito como materna, pelo menos para o município.

Por outro lado, a alta porcentagem de subnotificação das mortes por complicações do aborto suscita algumas hipóteses ou especulações relacionadas tanto ao comportamento da mulher diante da situação de um aborto provocado, quanto do profissional médico que individualmente falha no diagnóstico ou, por razões legais, deixa de emitir na declaração de óbito a causa básica da morte (aborto), temendo algum envolvimento legal. É possivel, ainda, aventar falha institucional, como nos serviços de verificação de óbitos que não têm, pelo menos no município, praticado de maneira 
sistemática a abertura do útero em casos de morte de mulheres em idade reprodutiva.

Alguns casos levantados podem ilustrar melhor este painel. Primeiro, o de uma mulher de 28 anos, casada. Na história clínica e nas evoluções durante o período de internação, não constam relatos sobre gravidez. A causa de morte atestada na declaração de óbito foi broncopneumonia bilateral. O laudo necroscópico descreve presença de oclusão trombótica de vasos pulmonares, infarto pulmonar, broncopneumonia e útero pós-aborto com endometrite aguda purulenta. Esta mulher parece não ter "informado" em nenhum momento de sua internação que tivesse praticado um aborto e, assim, não foi sequer realizado um exame ginecológico.

Um segundo caso, de mulher de 33 anos, casada. A DO, emitida pelo SVO (Serviço de Verificação de Óbito), atesta como causa da morte infarto agudo do miocárdio. Identificou-se o prontuário clínico do hospital onde foi prestado o primeiro atendimento e onde a mulher permaneceu por algumas horas, antes de sua morte. Na história clínica houve relato de aborto provocado em gestação de dois meses, três dias antes do atendimento. O exame clínico, naquele momento, revelava estado séptico e insuficiência cardiaca congestiva. Em laudo ecográfico realizado ainda em vida, constava diagnóstico de conteúdo intra-uterino, sugestivo de restos ovulares. Como na DO não constasse nenhuma informação, além de infarto agudo do miocárdio, concluiu-se que no SVO o útero não deve ter sido examinado. Estes exemplos, provavelmente, refletem a maior parte dos casos de morte materna por aborto ocorridos no Brasil e que têm sido, ao longo dos anos, negligenciados.

A discussão sobre programas de intervenção para redução da mortaldade materna, principalmente em países em desenvolvimento, passa pela necessidade de se reduzir o risco de morte secundária ao aborto inseguro ${ }^{17}$. A prevenção da gravidez indesejada, por meio da garantia de métodos contraceptivos a toda mulher em idade reprodutiva associada a programas de orientação sobre anticoncepção, inclusive pelos agentes de saúde, pode ser o ponto de partida para a redução da mortalidade materna por aborto. Contudo, podem não ser suficientes para a redução da gravidez indesejada, ao passo que as sanções legais, culturais e/ou religiosas não têm se mostrado efetivas para reduzir o número de abortos provocados.

As mulheres de baixo nivel sócio-cultural e econômico são, sem dúvida, aquelas que mais se ressentem desta condição, uma vez que ao grupo social mais favorecido é possivel obter o aborto de maneira segura, com minimização dos riscos. Em nossos casos, embora isso não seja possivel de se confirmar com números, as mulheres que morreram por uma complicação do aborto, em sua maioria, não possuiam convênio de saúde, condição que em nosso meio indiretamente relaciona-se a menor nível sócio-econômico e cultural.

No Brasil a indução do aborto é ilegal e, segundo o Código Penal vigente desde 1940, considerado "um crime contra a vida". É permitido em apenas duas situações: nas condições em que a evolução da gravidez traduz-se em risco de vida para a mãe ou quando a gravidez é fruto de estupro, devendo sua prática ser precedida pelo consentimento da mulher. Embora na última década tenham-se retomado as discussões sobre aborto pelo Congresso Nacional, com o apoio de grupos feministas e religiosas, os progressos alcançados ainda são mínimos ${ }^{18}$.

O segundo maior índice de subnotificação recaiu no grupo de causas obstétricas indiretas, com $66,6 \%$ ou $2 / 3$ casos, para o período de estudo. Esta situação chega a ser comparável à subnotificação de centros desenvolvidos, como a Holanda, onde o subregistro de morte materna por causa obstétrica indireta foi de $56 \%$ ou $25 / 45$ casos, no período de 1983 a $1992^{8}$. A condição de uma doença de base na mulher, levando ao óbito ocorrido principalmente no puerpério, sem uma relação aparente com a gravidez, pode ser um fator colaborador que atenua a falha do médico em registrar na DO o estado grávido puerperal, embora não se justifique.

Dentre as causas indiretas de morte materna, a hemorragia intracraniana, secundária à rotura de aneurisma cerebral, confirmada por necrópsia, correspondeu à totalidade dos casos. Esta condição pode ser confundida com AVCH, secundária a crise hipertensiva ou a eclâmpsia e, nestes casos, seriam mortes de causa obstétrica direta. Tal situação confirma a necessidade de realização de necrópsia também em mortes maternas classificadas originalmente como diretas. Das subnotificações encontradas na Holanda, a hemorragia cerebral também esteve associada à maior porcentagem de casos, com $67 \%$ ou $12 / 18$ casos.

Estes resultados vêm confirmar a necessidade de implantação de real vigilância epidemiológica da morte materna, bem como reforçar a relevância do risco de morte materna por aborto, além do aprofundamento das discussões, tanto legais quanto técnicas, sobre a maior efetividade dos programas de prevenção da morte materna. 


\section{SUMMARY}

Purpose: to identify and quantify the underreporting of maternal mortality, from death certificates (DC) in Campinas, São Paulo, from 1992 to 1994.

Methods: a total of 216 DC whose causes of death were maternal (declared and/or presumed) were selected among the 1032 DC of 10 to 49-year-old women. A complementary investigation was performed on hospital records, at the death verifying units, and in households.

Results: eight additional maternal deaths were identified among the 204 DC with presumed maternal death. This corresponded to an underreporting rate of $40 \%$ or to a correction factor of 1.67 for the official MMR. The first cause of underreporting was abortion (71.5\% or 05/07) and indirect maternal deaths represented the second cause (66.6\% or 02/ 03).

Conclusions: the death certificate cannot be considered the only source to identify maternal death. Complementary investigation of the presumable causes of maternal death should be performed. Legislation, social and religious factors might influence the underreporting of abortion as the cause of maternal death.

KEY WORDS: Maternal mortality. Abortion. Reproductive health.

\section{Referências}

1. Herz B, Measham AR. La iniciativa de la maternidad segura. Propuestas de acción. Washington, DC: Banco Mundial; 1987.

2. WHO/UNICEF. Revised 1990 estimates of maternal mortality: a new approach by WHO and UNICEF. Geneva: WHO/UNICEF; 1996. (WHO/FRH/ MSM/96.11;UNICEF/PLN/96.1).

3. Laurenti R, Buchalla CM, Lolio CA, Santo AH, Mello Jorge MHP. Mortalidade de mulheres em idade fértil no municipio de São Paulo (Brasil), 1986. I Metodologia e resultados gerais. Rev Saúde Públ 1990; 24:128-33.

4. Cecatti JG, Faúndes A, Surita FGC. Maternal mortality in Campinas: evolution, underregistration and avoidance. São Paulo Med J 1999;117:5-12.

5. Laurenti R, Mello Jorge MHP. O atestado de óbito. $2^{a}$ ed. São Paulo: Centro da OMS para a Classificação da Doença de Chagas; 1987.
6. Albuquerque RM, Cecatti JG, Hardy E, Faúndes A. Mortalidade materna em Recife 1. Avaliação da subenumeração de estatísticas oficiais. Cad Saúde Públ 1997; 13:59-65.

7. Paxman JM, Rizo A, Brown L, Benson J. The clandestine epidemic: the practice on unsafe abortion in Latin America. Stud Fam Plann 1993; 24:205-26

8. Schuitemaker NW, Gravenhorst JB, Van Geijn HP, Dekker GA, Van Dongen PW. Maternal mortality and its prevention. Eur J Obstet Gynecol Reprod Biol 1991; 42 (Suppl):S31-5.

9. Costa A. Direitos reprodutivos: riscos e encruzilhadas. Brasília; 1993. (Mimeografado).

10.Braga LFCO. Aborto, anticoncepção e mortalidade materna. In: Faúndes A, Cecatti JG, editores. Mortalidade materna uma tragédia evitável. Campinas: Ed. UNICAMP; 1991. p.139-50.

11.Benedetti TJ, Starzyk P, Frost F. Maternal deaths in Washington State. Obstet Gynecol 1985; 66:99-101.

12.Bouvier-Colle MH, Varnoux N, Costes P, Hatton F. Reasons for the under-reporting of maternal mortality in France, as indicated by a survey of all deaths among women of childbearing age. Int J Epidemiol 1991; 20:717-21.

13.United Kingdom. Department of Health. Report on confidential enquires into maternal deaths in the United Kingdom 1988/1990. London: HMSO; 1994.

14.Parpinelli MA, Faúndes A, Surita FGC, Pereira BG, Cecatti JG. Mortalidade materna na cidade de Campinas, no período de 1992 a 1994. Rev Bras Ginecol Obstet 1999;21:227-32.

15.Organização Mundial de Saúde. Classificação Estatística Internacional de Doenças e Problemas Relacionados à Saúde, $10^{\mathrm{a}}$ revisão. São Paulo: Centro Colaborador da OMS para Classificação de Doenças em Português; 1995. v.1.

16.Pazero LC, Marcus PAF, Vega CEP, Boyaciyan K, Barbosa AS. Estudo da mortalidade materna no município de São Paulo durante o ano de 1996. Rev Bras Ginecol Obstet 1998;20:395-403.

17.Faúndes A, Cecatti JG, Bacha AM, Pinotti JA. Interventions to reduce maternal mortality. Rev Paul Med 1989;107:47-52.

18. Hardy E, Rebello I. La discusión sobre el aborto provocado en el Congreso Nacional Brasileño: el papel del movimiento de mujeres. Cad Saúde Públ 1996; 12:259-66. 